This item was submitted to Loughborough's Research Repository by the author.

Items in Figshare are protected by copyright, with all rights reserved, unless otherwise indicated.

\title{
A highly selective synthesis of the indolo[2,3-a]quinolizine ring system and application to natural product synthesis
}

PLEASE CITE THE PUBLISHED VERSION

https://doi.org/10.1002/ejoc.200500412

PUBLISHER

(c) Wiley-VCH Verlag

LICENCE

CC BY-NC-ND 4.0

\section{REPOSITORY RECORD}

Allin, Steven M., Christopher I. Thomas, James E. Allard, Kevin J. Doyle, and Mark R.J. Elsegood. 2019. "A Highly Selective Synthesis of the Indolo[2,3-a]quinolizine Ring System and Application to Natural Product Synthesis". figshare. https://hdl.handle.net/2134/654. 
This item was submitted to Loughborough's Institutional Repository by the author and is made available under the following Creative Commons Licence conditions.

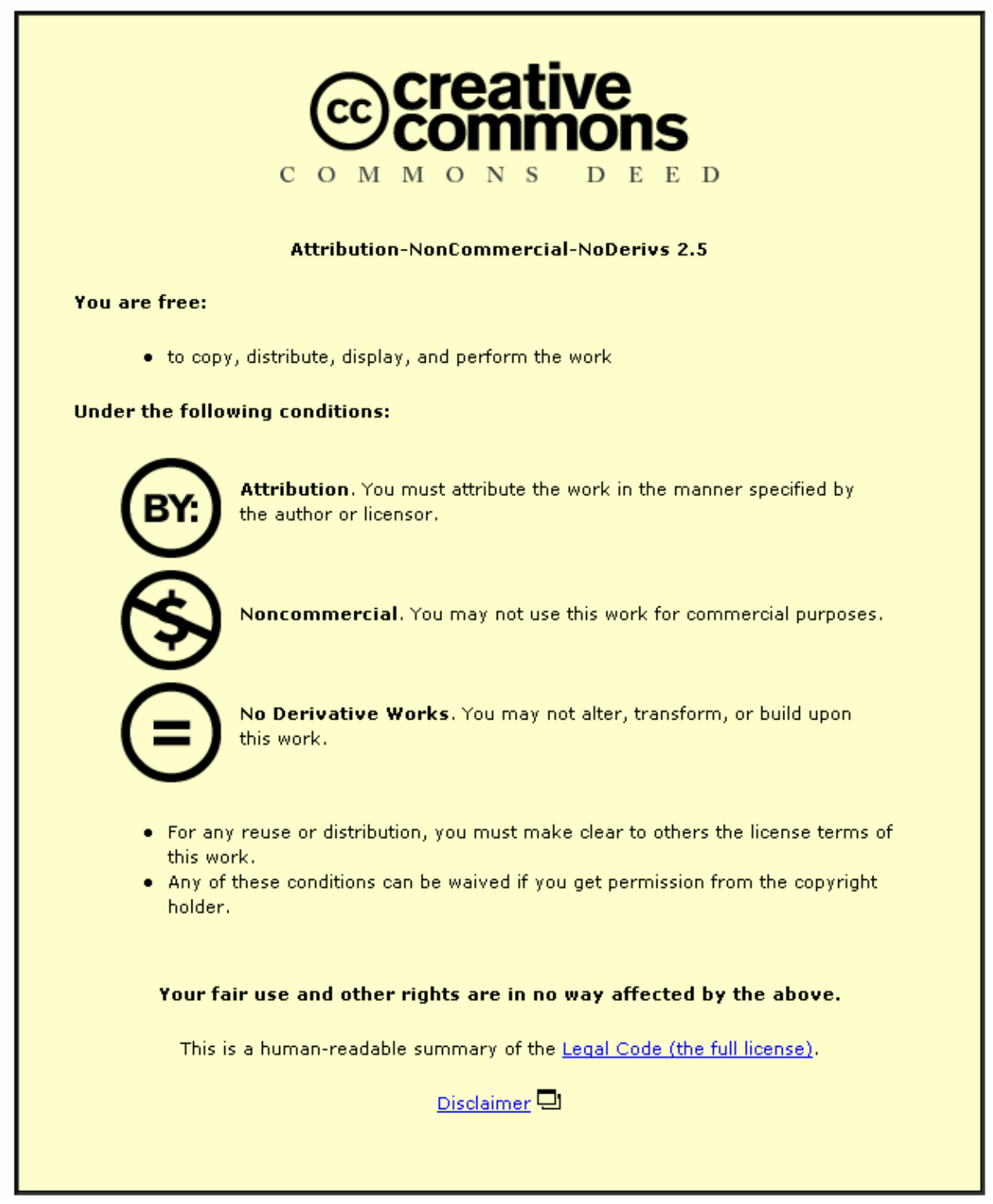

For the full text of this licence, please go to: http://creativecommons.org/licenses/by-nc-nd/2.5/ 


\title{
L Loughborough University
}

A Highly Stereoselective Synthesis of the

Indolo[2,3-a]quinolizine Ring System and Application to

Natural Product Synthesis

\section{[EUROPEAN J OURNAL OF ORGANI C CHEMI STRY (19): 4179-4186 SEP 26 2005]}

Steven M. Allin, ${ }^{\text {a* }}$ Christopher I. Thomas, James E. Allard, ${ }^{\text {a }}$

Kevin Doyle $^{\mathrm{b}}$ and Mark R. J. Elsegood ${ }^{\mathrm{a}}$

\section{${ }^{a}$ Department of Chemistry, Loughborough University, Loughborough, Leicestershire, LE11 3TU, England ${ }^{b}$ OSI Pharmaceuticals, Watlington Road, Oxford, OX4 6LT, England}

Key Words: Natural products, Heterocycles, Lactams, Asymmetric Synthesis

\begin{abstract}
We present a facile and highly stereoselective approach to the indolo[2,3-a]quinolizine ring system from a readily available, non-racemic chiral template. We demonstrate the potential for application of this methodology to natural product synthesis through conversion of the template to some representative indole alkaloids with high enantiomeric purity in both enantiomeric series.
\end{abstract}

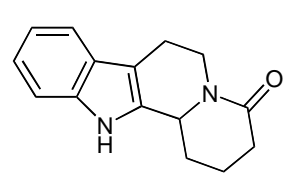

1

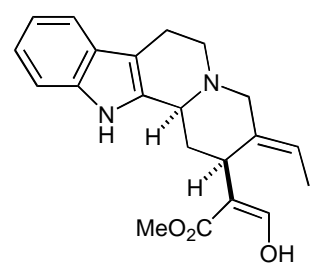

2

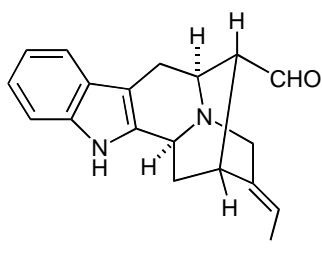

3

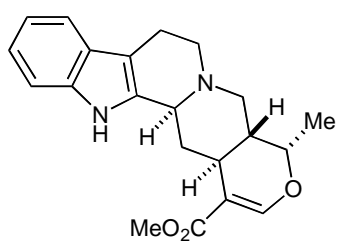

4

${ }^{*}$ Corresponding author. S.M.Allin@lboro.ac.uk 
Figure 1

The indolo[2,3-a]quinolizine ring system $\mathbf{1}$ is of great interest and significance since this heterocyclic template is found within a plethora of highly bioactive indole alkaloids, including geissoschizine $2,{ }^{[1]}$ vellosimine $3^{[2]}$ and ajmalicine $4 .{ }^{[3]}$ The presence of the lactam carbonyl in templates such as $\mathbf{1}$ would allow for possible further functionalisation en route to the natural product targets. Recent approaches to the construction of this heterocyclic target system by other groups have included the diastereoselective vinylogous Mannich reaction, ${ }^{[4]}$ BischlerNapieralski reaction, ${ }^{[5]}$ Fischer indole synthesis ${ }^{[6]}$ and the asymmetric Pictet-Spengler reaction. ${ }^{[7]}$

We have recently developed a new and general approach for the stereoselective synthesis of a range of non-racemic heterocycles that involves the cyclisation of pendent aromatic substituents onto $\mathrm{N}$-acyliminium intermediates as the key ring-forming step. ${ }^{[8]}$ Based on our novel approach to the indolizino[8,7-b]indole ring system, ${ }^{[8]}$ we recognised that a suitably substituted bicyclic lactam could act as a precursor in a stereoselective approach to the indolo[2,3-a]quinolizine ring system.

Our approach to the synthesis of the required bicyclic lactam substrate 5 followed the general method previously used in our group. ${ }^{[8]}$ The $\beta$-amino alcohol derivative of $(S)$-tryptophan was reacted under Dean-Stark conditions in toluene with an appropriate functionalised substrate for $48 \mathrm{~h}$ (Scheme 1). Under these reaction conditions we were able to isolate the expected bicyclic lactam in $69 \%$ yield as a 5:1 mixture of separable diastereoisomers, $5 \mathbf{a}$ and $\mathbf{5 b}$.

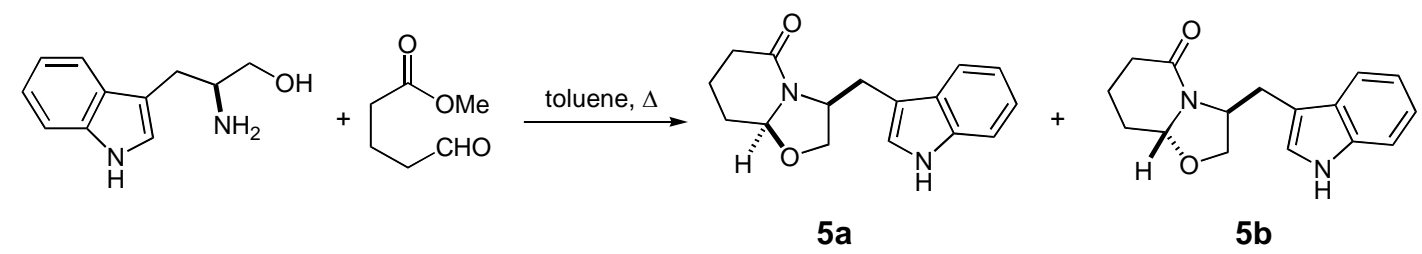

Scheme 1

The relative stereochemistry of the major diastereoisomer 5a was determined by single crystal X-ray analysis. ${ }^{[9]}$ This indole-containing bicyclic lactam is a novel example of the 
fused 5,6-ring system favoured by Amat and Bosch, ${ }^{[10]}$ and the relative stereochemistry observed for the major isomer $\mathbf{5 a}$ is consistent with results obtained both by these researchers and in our own previous work in other areas. ${ }^{[8 b]}$

In a previous communication we noted briefly that treatment of the initial mixture of diastereoisomers of substrate 5 with $\mathrm{TiCl}_{4}$ gave the desired indolo[2,3-a]quinolizine target $\mathbf{6}$ in $54 \%$ yield, but with only a poor level of product diastereoselectivity $(5: 2) .{ }^{[8 a]}$ We have since discovered that simply treating the mixture of bicyclic lactam substrate diastereoisomers, 5a and $5 \mathbf{b}$, with $2 \mathrm{M} \mathrm{HCl}$ in ethanol at room temperature for 20 hours gives an excellent yield of $95 \%$ for the cyclisation reaction, and leads to the formation of the desired indolo[2,3a]quinolizine product as a single diastereoisomer (Scheme 2).

The relative stereochemistry of the single diastereoisomer 6 was determined by single crystal $\mathrm{X}$-ray analysis (Figure 2) ${ }^{[9]}$ and was found to be as favoured in the $\mathrm{TiCl}_{4}$ mediated cyclisation reaction that had previously given only a 5:2 ratio of product diastereoisomers.<smiles>O=C1CCC[C@H]2OC[C@@H](Cc3c[nH]c4ccccc34)N12</smiles>

$5 a, b$

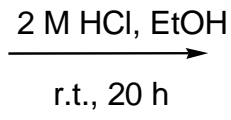

Scheme 2

To highlight the synthetic utility of this methodology in the target synthesis of complex indole alkaloids and their synthetic analogues, we undertook the synthesis of a simple indole alkaloid, (S)-(-)-1,2,3,4,6,7,12,12b-octahydroindolo[2,3-a]quinolizine, 11, the main constituent of Dracontomelum mangiferum B1. ${ }^{[1]}$ In order to access the natural $(S)$-enantiomer of the target we were required to work with the enantiomer of the template. Hence compound 7 was prepared as a single diastereoisomer from $(R)$-tryptophan by analogous chemistry to that 
described above. Our synthetic route to the natural product $\mathbf{1 1}$ from $\mathbf{7}$ is highlighted in Scheme 3.

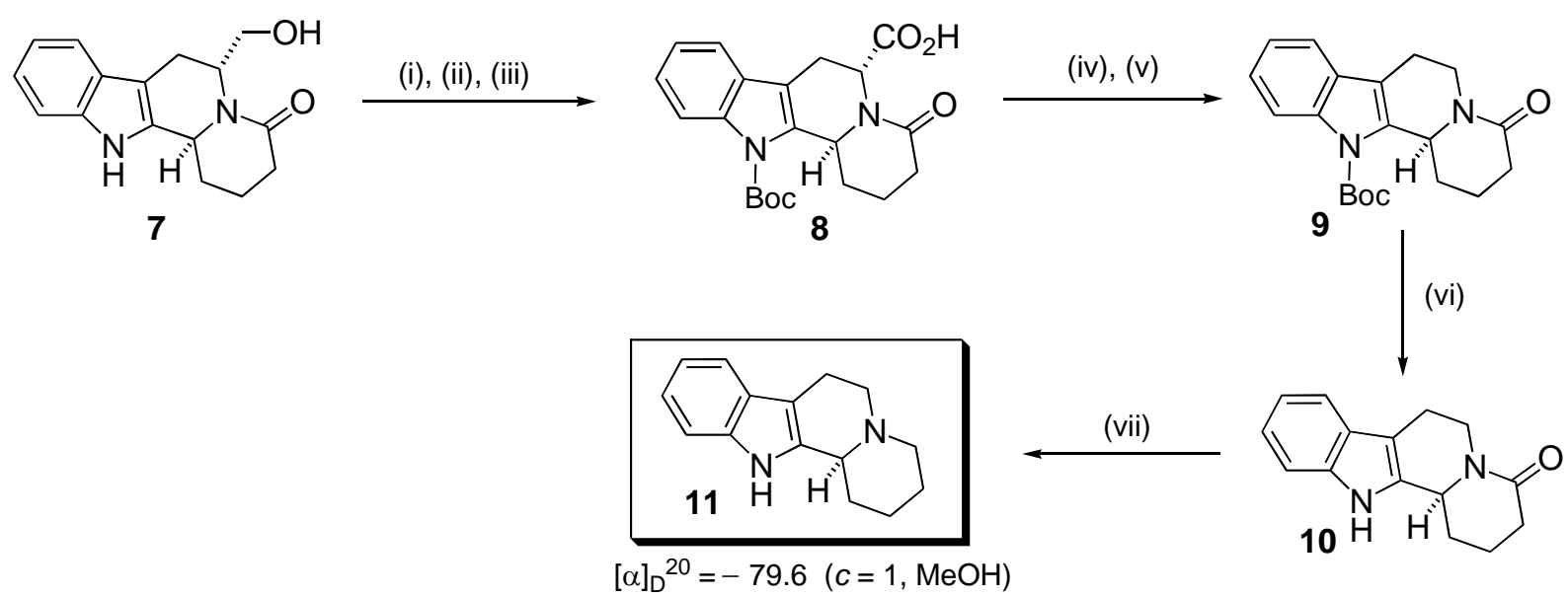

Scheme 3. (i) IBX, DMSO, r.t., 24 h (60\%); (ii) $\mathrm{Et}_{3} \mathrm{~N}$, (Boc) $)_{2} \mathrm{O}$, DMAP, THF, r.t., 4 h (63\%); (iii) $\mathrm{NaClO}_{2}$, $\mathrm{NaH}_{2} \mathrm{PO}_{4}, 1$-methyl-1-cyclohexene, $\mathrm{CH}_{3} \mathrm{CN}, t$-BuOH, $\mathrm{H}_{2} \mathrm{O}, 0{ }^{\circ} \mathrm{C}$ to r.t., $18 \mathrm{~h}$ (70\%); (iv) ( $\left.\mathrm{PhSe}\right)_{2}, \mathrm{PBu}_{3}, \mathrm{CH}_{2} \mathrm{Cl}_{2}$, $0{ }^{\circ} \mathrm{C}$ to r.t., $18 \mathrm{~h}$ (66\%); (v) $n$-Bu $\mathrm{Bu}_{3} \mathrm{SnH}$, AIBN, toluene, $80{ }^{\circ} \mathrm{C}, 2 \mathrm{~h}(98 \%)$; (vi) TBAF, THF, $\Delta, 9 \mathrm{~h}(63 \%)$; (vii) $\mathrm{LiAlH}_{4}, \mathrm{THF}, \Delta, 3 \mathrm{~h}$ then r.t., $12 \mathrm{~h}(96 \%)$.

Our previous method to remove the hydroxymethyl group from templates such as 7 has involved a rhodium-induced decarbonylation sequence. ${ }^{[8]}$ Due to the rather long reaction times generally needed for our substrates in this protocol we have now applied an easier approach that relies upon a decarboxylation strategy. Compound 7 was oxidised to the carboxylic acid derivative 8 through the corresponding aldehyde; from $\mathbf{8}$ we generated the acyl selenide derivative and subsequently performed a tin-mediated deacylation to yield the indolo[2,3-a]quinolizine ring system 9. Deprotection of the indole nitrogen gave known compound $(S)-\mathbf{1 0}$ in $>95 \%$ e.e. by comparison of optical rotation data. ${ }^{[12 \mathrm{a}]}$ Reductive removal of the lactam carbonyl group completed the synthesis of the natural product. Target $(S)-(-)-\mathbf{1 1}$ was found to have an e.e. of $95 \%$ and the same absolute configuration as the natural product by comparison of optical rotation data. ${ }^{[12 b]}$ 
In order to further extend the application of our methodology we chose to pursue the synthesis of deplancheine, 14, an alkaloid isolated from the New Caledonian plant Alstonia deplanchei. ${ }^{[13]}$ As a consequence of the work of Meyers in 1986 that described an asymmetric approach to deplancheine, the absolute configuration of the natural product was determined to be $R^{[13 \mathrm{a}]}$

A successful synthesis of deplancheine would require us to further functionalise the lactam ring of the template. Construction of the requisite ethylidene moiety was achieved through a three-step procedure involving generation of the lithium enolate from $(R)-\mathbf{9}$, prepared by analogous chemistry to that described in Scheme 3 but starting from $(S)$-tryptophan. Subsequent aldol reaction with acetaldehyde, activation of the hydroxyl group by mesylation and finally DBN-induced elimination gave the initial target 12 (Scheme 4). We found that whereas a THF/DCM solvent mixture in the elimination step gave only a 4.7:1 ratio in favour of the desired $E$-isomer 12, if the elimination procedure was carried out in THF alone as solvent the $E$-regioisomer of the ethylidene product 12 was obtained exclusively. ${ }^{[14]}$ TBAFinduced deprotection of the indole nitrogen atom liberated the lactam intermediate $13 .{ }^{[15]}$

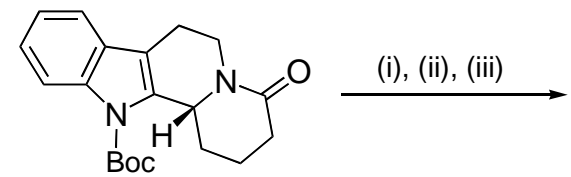

R-9

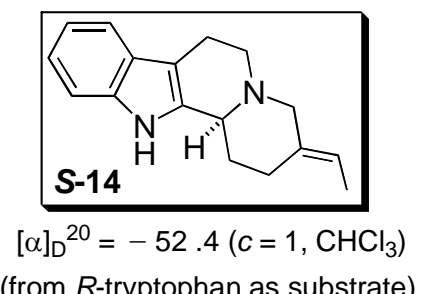

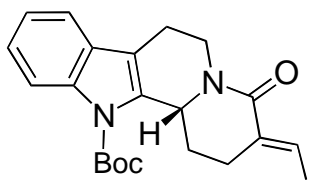

12

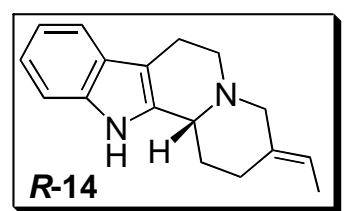

$[\alpha]_{D}^{20}=+52.0\left(c=1, \mathrm{CHCl}_{3}\right)$

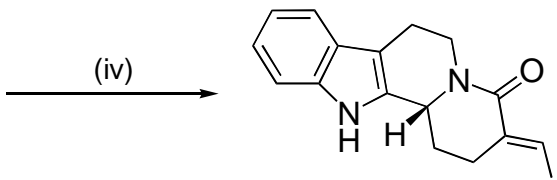

13

\section{(v), (vi)}

Scheme 4. Asymmetric Synthesis of Deplancheine: (i) $\mathrm{LDA}, \mathrm{CH}_{3} \mathrm{CHO}, \mathrm{THF},-78{ }^{\circ} \mathrm{C}$ to r.t., 24 h; (ii) $\mathrm{Et}{ }_{3} \mathrm{~N}$, $\mathrm{MsCl}, \mathrm{DCM},-40{ }^{\circ} \mathrm{C}$ to r.t., $3 \mathrm{~h}$; (iii) DBN, THF, r.t., $16 \mathrm{~h}$ (65\% for 3 steps); (iv) TBAF, THF, $\Delta, 9 \mathrm{~h}(63 \%)$; (v) $\mathrm{Me}_{3} \mathrm{OBF}_{4}$, 2,6-di-t-Bu-Py, DCM, r.t., 21 h; (vi) $\mathrm{NaBH}_{4}, \mathrm{MeOH}, 0{ }^{\circ} \mathrm{C}, 0.5 \mathrm{~h}$ (77\% for 2 steps). 
The synthesis of $(R)$-deplancheine was completed through removal of the lactam carbonyl group as described in Scheme 4 through application of a route described by Martin and coworkers. ${ }^{[16]}$ The desired target, $(R)$-(+)-deplancheine, 14, was obtained with an e.e. of $>95 \%$ and the same absolute configuration as the natural product by comparison of optical rotation data. ${ }^{[13 a]}$ An X-ray crystal structure of the product $R-\mathbf{1 4}$ was obtained and is presented Figure 2.

The crystals were obtained by slow evaporation of a dichloromethane solution of the compound. The asymmetric unit comprises two molecules of $\mathbf{1 4}$ and one molecule of water. The water molecule links together three molecules of $\mathbf{1 4}$ via strong $\mathrm{H}$-bonds, while the $\mathrm{NH}$ on $\mathrm{N}(27)$ forms a weak $\mathrm{N}-\mathrm{H} \cdots \pi$ interaction to the ring $\mathrm{C}(22 \mathrm{~B})>\mathrm{C}(26)$ on a symmetry related molecule.

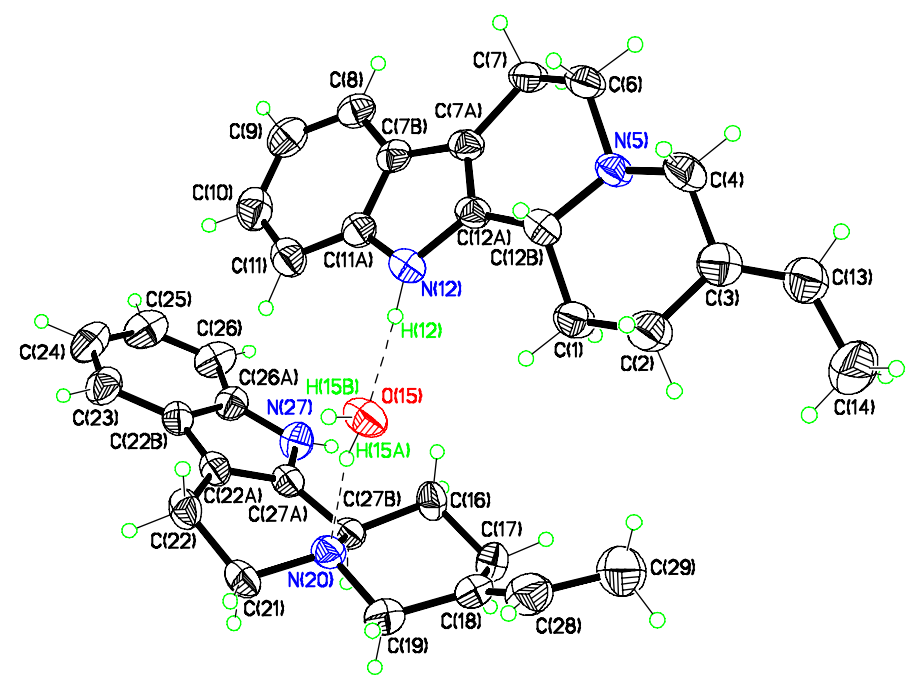

Figure 2.

To demonstrate the potential synthetic utility of our methodology we have also undertaken an asymmetric synthesis of the enantiomer of deplancheine. Our route mirrors that described in Scheme 4 , but uses the $(R)$-enantiomer of tryptophan as the starting material. The desired target, $(S)-(-)$-deplancheine (Scheme 4 ), was isolated with an e.e. of $>95 \%$ by comparison of optical rotation data. ${ }^{[13 a]}$ 
In summary, we report a facile and highly stereoselective approach to the important indolo[2,3-a]quinolizine template from readily available non-racemic substrates, and have demonstrated the structural modification of the template to deliver indole alkaloids with high enantiomeric purity in both enantiomeric series. Current work is focused on extending the methodology described in this paper to other, more complex indole alkaloid targets. Our progress will be reported in due course.

Acknowledgements: Loughborough University and OSI Pharmaceuticals (joint studentship to CIT), EPSRC (Quota studentship to JA).

\section{Experimental Section}

\section{General information}

\section{Solvents and Reagents}

All solvents where necessary were dried, distilled and stored over $4 \AA$ molecular sieves prior to use.

\section{Chromatographic procedures}

Analytical thin layer chromatography (TLC) was carried out using aluminium-backed plates coated with $0.2 \mathrm{~mm}$ silica. Plates were visualised under UV light (at $254 \mathrm{~nm}$ ) or by staining with either potassium permanganate solution or PMA (phosphomolybdic acid). Flash column chromatography was carried out using Merck Kieselgel (70-230 Mesh ASTM). Samples were applied as saturated solutions in an appropriate solvent or pre-adsorbed onto the minimum quantity of silica. Hand bellows were used to apply pressure when required at the column.

\section{Spectra}

IR spectra were recorded in the range from 4000 to $600 \mathrm{~cm}^{-1}$. Solid samples were run as nujol 
mull, and liquids as thin films.

Nuclear Magnetic Resonance (NMR) spectra: multiplicities were recorded as broad peaks (br), singlets (s), doublets (d), triplets $(\mathrm{t})$, double doublets (dd) and multiplets (m). All NMR samples were made up in deuterated chloroform with all values quoted in ppm relative to tetramethylsilane as internal reference. Coupling constants ( $J$ values) are reported in Hertz $(\mathrm{Hz})$. Diastereoisomer ratios were calculated from the integration of suitable peaks in the proton NMR.

\section{Experimental Procedures}

\section{3-([1H-Indol-3-yl]methyl)hexahydrooxazolo[3,2-a][1,3]pyridin-5-one, 5}

(2S)-2-Amino-3-(1H-indol-3-yl)propan-1-ol (3.0 g, $15.8 \mathrm{mmol})$ and methyl-5-oxopentanoate $(2.1 \mathrm{~g}, 15.8 \mathrm{mmol})$ were added to toluene $(150 \mathrm{ml})$ and refluxed under Dean-Stark conditions for 48 hours. The mixture was cooled to room temperature and the solvent removed under reduced pressure to yield the target compound. $250 \mathrm{MHz}{ }^{1} \mathrm{H}$ NMR spectroscopy on the crude product mixture revealed the formation of 5 as a 5:1 mixture of diastereoisomers. These diastereoisomers were purified and separated by flash column chromatography over silica using ethyl acetate as eluent (2.90 g, 69\%).

Major isomer (3S,8aS)-5a isolated as a yellow solid which was recrystallized from dichloromethane to yield colourless needles; Mp 152-156 ${ }^{\circ} \mathrm{C} ;[\alpha]_{\mathrm{D}}=-39.1\left(c=1.7, \mathrm{CH}_{2} \mathrm{Cl}_{2}\right)$; (Found: C, 71.04; H, 6.69; N, 10.27; $\mathrm{C}_{16} \mathrm{H}_{18} \mathrm{~N}_{2} \mathrm{O}_{2}$ requires $\mathrm{C}, 71.09 ; \mathrm{H}, 6.71 ; \mathrm{N}, 10.36 \%$ ); $v_{\max }\left(\right.$ thin film, DCM)/cm $3274(\mathrm{NH}), 1628(\mathrm{NC}=\mathrm{O}) ; \delta_{\mathrm{H}}\left(400 \mathrm{MHz} ; \mathrm{CDCl}_{3}\right) 1.43-1.57(1 \mathrm{H}$, m), 1.67-1.77 (1H, m), 1.96-2.01 (1H, m), 2.24-2.28 (1H, m), 2.41-2.45 (2H, m), $2.68(1 \mathrm{H}$, dd, $J 16,8), 3.67-3.76(2 \mathrm{H}, \mathrm{m}), 4.03(1 \mathrm{H}, \mathrm{d}, J$ 8), 4.28-4.33 (1H, m), $4.68(1 \mathrm{H}, \mathrm{dd}, J 12,4)$, $7.03(1 \mathrm{H}, \mathrm{d}, J 4), 7.05-7.22(2 \mathrm{H}, \mathrm{m}), 7.36(1 \mathrm{H}, \mathrm{d}, J 8), 7.82(1 \mathrm{H}, \mathrm{d}, J 8), 8.24(1 \mathrm{H}, \mathrm{br}, \mathrm{s})$; $\delta_{\mathrm{C}}\left(100 \mathrm{MHz} ; \mathrm{CDCl}_{3}\right) 17.6\left(\mathrm{CH}_{2}\right), 27.0\left(\mathrm{CH}_{2}\right), 28.4\left(\mathrm{CH}_{2}\right), 31.1\left(\mathrm{CH}_{2}\right), 56.1(\mathrm{CH}), 70.0\left(\mathrm{CH}_{2}\right)$, $89.0(\mathrm{CH}), 111.1(\mathrm{CH}), 112.6(\mathrm{C}), 119.4(\mathrm{CH}), 119.6(\mathrm{CH}), 122.2(\mathrm{CH}), 122.4(\mathrm{CH}), 127.7$ 
(C), $136.2(C), 168.1\left(\mathrm{NC}=\mathrm{O}\right.$ ); $\mathrm{MS}$ (EI) $\mathrm{m} / z 270\left[\mathrm{M}^{+}, 100 \%\right]$ (Found: $\mathrm{M}^{+}, 270.13690$. $\mathrm{C}_{16} \mathrm{H}_{18} \mathrm{~N}_{2} \mathrm{O}_{2}$ requires 270.13683).

Minor isomer (3S,8aR)-5b isolated as a pale yellow oil; $[\alpha]_{\mathrm{D}}=+14.3\left(c=2.0, \mathrm{CH}_{2} \mathrm{Cl}_{2}\right)$ (Found: $\mathrm{C}$, 70.97; H, 6.69; N, 10.35; $\mathrm{C}_{16} \mathrm{H}_{18} \mathrm{~N}_{2} \mathrm{O}_{2}$ requires $\mathrm{C}, 71.09 ; \mathrm{H}, 6.71 ; \mathrm{N}, 10.36 \%$ ); $v_{\max }\left(\right.$ thin film, DCM)/cm $3279(\mathrm{NH}), 1628(\mathrm{NC}=\mathrm{O}) ; \delta_{\mathrm{H}}\left(400 \mathrm{MHz} ; \mathrm{CDCl}_{3}\right) 1.33-1.42(1 \mathrm{H}$, m, $\left.\mathrm{CH}(\mathrm{H}) \mathrm{CH}_{2} \mathrm{CH}_{2} \mathrm{C}=\mathrm{O}\right), 1.51-1.59\left(1 \mathrm{H}, \mathrm{m}, \mathrm{CH}_{2} \mathrm{CH}(\mathrm{H}) \mathrm{CH}_{2} \mathrm{C}=\mathrm{O}\right), 1.80-1.88 \quad(1 \mathrm{H}, \mathrm{m}$, $\left.\mathrm{CH}_{2} \mathrm{CH}(\mathrm{H}) \mathrm{CH}_{2} \mathrm{C}=\mathrm{O}\right), \quad 2.14-2.18 \quad\left(1 \mathrm{H}, \quad \mathrm{m}, \quad \mathrm{CH}(\mathrm{H}) \mathrm{CH}_{2} \mathrm{CH}_{2} \mathrm{C}=\mathrm{O}\right), \quad 2.27-2.37 \quad(1 \mathrm{H}, \mathrm{m}$, $\mathrm{C} H(\mathrm{H}) \mathrm{C}=\mathrm{O}), 2.53(1 \mathrm{H}, \mathrm{dd}, J 16,8, \mathrm{CH}(\mathrm{H}) \mathrm{C}=\mathrm{O}), 3.05(1 \mathrm{H}, \mathrm{dd}, J 16,8, \mathrm{CH}(\mathrm{H}) \mathrm{C}=\mathrm{C}), 3.32(1 \mathrm{H}$, dd, $J 16,4, \mathrm{CH}(\mathrm{H}) \mathrm{C}=\mathrm{C}), 3.69(1 \mathrm{H}, \mathrm{dd}, J 8.8,7.2, \mathrm{CH}(\mathrm{H}) \mathrm{O}), 4.04-4.09(1 \mathrm{H}, \mathrm{m}, \mathrm{CH}(\mathrm{H}) \mathrm{O}), 4.46$ $\left(1 \mathrm{H}, \mathrm{dd}, J 8,4, \mathrm{NCHOCH}_{2}\right), 4.60-4.67\left(1 \mathrm{H}, \mathrm{m}, \mathrm{NCHCH}_{2} \mathrm{O}\right), 7.01(1 \mathrm{H}, \mathrm{d}, J 4, \mathrm{C}=\mathrm{CHNH})$, 7.10-7.14 (1H, m, ArH), 7.17-7.21 (1H, m, ArH), $7.36(1 \mathrm{H}, \mathrm{d}, J 8, \operatorname{Ar} H), 7.69(1 \mathrm{H}, \mathrm{d}, J 8$, $\mathrm{ArH}), 8.34(1 \mathrm{H}, \mathrm{br}, \mathrm{s}, \mathrm{NH}) ; \delta_{\mathrm{C}}\left(100 \mathrm{MHz} ; \mathrm{CDCl}_{3}\right) 14.2\left(\mathrm{CH}_{2}\right), 27.6\left(\mathrm{CH}_{2}\right), 28.2\left(\mathrm{CH}_{2}\right), 31.4$ $\left(\mathrm{CH}_{2}\right), 54.5(\mathrm{CH}), 69.7\left(\mathrm{CH}_{2}\right), 87.3(\mathrm{CH}), 111.1(\mathrm{CH}), 111.2(\mathrm{C}), 119.2(\mathrm{CH}), 119.5(\mathrm{CH})$, $122.2(\mathrm{CH}), 122.6(\mathrm{CH}), 127.8(\mathrm{C}), 136.3(\mathrm{C}), 168.8(\mathrm{NC}=\mathrm{O})$; MS (EI) $m / z 270$ [M $\left.\mathrm{M}^{+}, 23.3 \%\right]$ (Found: $\mathrm{M}^{+}, 270.13690 . \mathrm{C}_{16} \mathrm{H}_{18} \mathrm{~N}_{2} \mathrm{O}_{2}$ requires 270.13683).

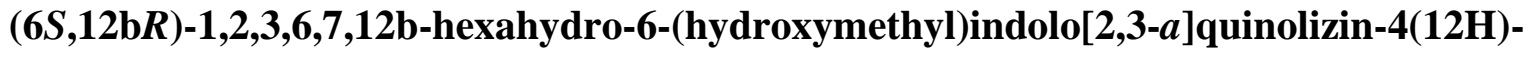 one, 6}

Compound 5a (3.1 g, $11.5 \mathrm{mmol})$ was dissolved in absolute ethanol (100 $\mathrm{ml})$ under a nitrogen atmosphere. The resulting solution was acidified to $\mathrm{pH} 1$ by the addition of $2 \mathrm{M} \mathrm{HCl}$ in ethanol and the mixture was stirred for a further 20 hours at room temperature. After this time the reaction was quenched by addition of saturated aqueous sodium bicarbonate and extracted with ethyl acetate $(3 \times 150 \mathrm{ml})$. The organic fraction was dried over anhydrous magnesium sulphate and the solvent removed by rotary evaporation to yield a light brown solid (2.9 $\mathrm{g}$, 95\%). $250 \mathrm{MHz}{ }^{1} \mathrm{H}$ NMR spectroscopy on the crude product mixture revealed formation of 6 as a single diastereoisomer, which was recrystallised from absolute ethanol to yield the target compound as colourless block-like crystals; Mp 288-290 ${ }^{\circ} \mathrm{C} ;[\alpha]_{\mathrm{D}}=+128.8(c=0.50, \mathrm{EtOH})$ (Found: C, 70.75; H, 6.67; N, 10.28; $\mathrm{C}_{16} \mathrm{H}_{18} \mathrm{~N}_{2} \mathrm{O}_{2}$ requires $\mathrm{C}, 71.09 ; \mathrm{H}, 6.71 ; \mathrm{N}, 10.36 \%$ ); 
$v_{\max }\left(\right.$ thin film, DCM)/cm $3277(\mathrm{NH}), 1618(\mathrm{NC}=\mathrm{O}) ; \delta_{\mathrm{H}}(400 \mathrm{MHz} ; \mathrm{DMSO}) 1.52-1.62(1 \mathrm{H}$, m), 1.73-1.86 (2H, m), 2.26-2.43 (2H, m), 2.59-2.63 (1H, m), 2.66 (1H, dd, $J 16,8), 2.80$ $(1 \mathrm{H}, \mathrm{d}, J 16), 3.35-3.37(2 \mathrm{H}, \mathrm{m}), 4.65(1 \mathrm{H}, \mathrm{d}, J 8), 4.81(1 \mathrm{H}, \mathrm{t}, J 4), 5.22(1 \mathrm{H}, \mathrm{q}, J 7.5), 6.95-$ $6.99(1 \mathrm{H}, \mathrm{m}), 7.04-7.08(1 \mathrm{H}, \mathrm{m}), 7.32\left(1 \mathrm{H}, \mathrm{d}, J\right.$ 8), 7.40 (1H, d, J 8), 10.09 (1H, br, s); $\delta_{\mathrm{C}}(100$ MHz; DMSO) $19.4\left(\mathrm{CH}_{2}\right), 21.0\left(\mathrm{CH}_{2}\right), 29.0\left(\mathrm{CH}_{2}\right), 32.7\left(\mathrm{CH}_{2}\right), 48.2(\mathrm{CH}), 50.6(\mathrm{CH}), 60.2$ $\left(\mathrm{CH}_{2}\right), 105.1(\mathrm{C}), 111.4(\mathrm{CH}), 118.1(\mathrm{CH}), 118.8(\mathrm{CH}), 121.3(\mathrm{CH}), 127.1(\mathrm{C}), 133.7(\mathrm{C})$, 136.7 (C), $168.8\left(\mathrm{NC}=\mathrm{O}\right.$ ); MS (EI) $m / z 270\left[\mathrm{M}^{+}, 100 \%\right]$ (Found: $\mathrm{M}^{+}, 270.13626 . \mathrm{C}_{16} \mathrm{H}_{18} \mathrm{~N}_{2} \mathrm{O}_{2}$ requires 270.13683 ).

\section{Three-step Route to Access 8:}

\section{(6R,12bS)-1,2,3,4,6,7,12,12b-octahydro-4-oxoindolo[2,3-a]quinolizine-6-carbaldehyde}

To a solution of 7 ( $5.0 \mathrm{~g}, 18.5 \mathrm{mmol})$ in dimethylsulfoxide $(40 \mathrm{ml})$ was added IBX (15.4 g, $55.5 \mathrm{mmol})$ with stirring under nitrogen. After 24 hours stirring at room temperature the solvent was removed and the resulting solid was re-suspended in ethyl acetate $(250 \mathrm{ml})$, before filtering through a small pad of celite. The filtrate was washed with water $(3 \times 250 \mathrm{ml})$, the organic phase was then dried with anhydrous magnesium sulphate, filtered and evaporated to dryness to yield the target compound which was purified by flash column chromatography over silica using ethyl acetate as eluent. The purified aldehyde was afforded as a pale yellow foam $(3.0 \mathrm{~g}, 60 \%)$; Mp 97.5-99 ${ }^{\circ} \mathrm{C} ;[\alpha]_{\mathrm{D}}=-201.3(c=1.2$,

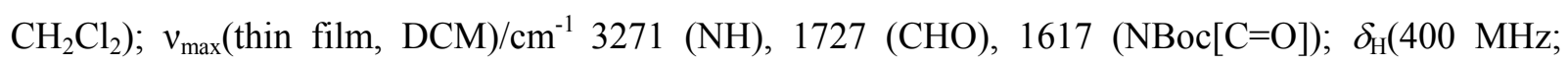
$\left.\mathrm{CDCl}_{3}\right)$ 1.66-1.77 (1H, m, $\left.\mathrm{CH}(\mathrm{H}) \mathrm{CH}_{2} \mathrm{CH}_{2} \mathrm{C}=\mathrm{O}\right), 1.99-2.05\left(2 \mathrm{H}, \mathrm{m}, \mathrm{CH}_{2} \mathrm{CH}_{2} \mathrm{C}=\mathrm{O}\right), 2.40-2.48(1 \mathrm{H}, \mathrm{m}$, $\left.\mathrm{C} H(\mathrm{H}) \mathrm{CH}_{2} \mathrm{CH}_{2} \mathrm{C}=\mathrm{O}\right), 2.49-2.54(1 \mathrm{H}, \mathrm{m}, \mathrm{CH}(\mathrm{H}) \mathrm{C}=\mathrm{O}), 2.69-2.76(1 \mathrm{H}, \mathrm{m}, \mathrm{CH}(\mathrm{H}) \mathrm{C}=\mathrm{O}), 3.10(1 \mathrm{H}, \mathrm{ddd}$, $J$ 16, 8, 4, CH(H)CHCHO), 3.41-3.47 (1H, m, CH(H)CHCHO), 4.89-4.93 (1H, m, C=CCH), 5.975.99 (1H, m, CHCHO), 7.11-7.24 (2H, m, ArH), 7.30-7.32 (1H, m, ArH), 7.53-7.55 (1H, m, ArH), $8.15(1 \mathrm{H}, \mathrm{br}, \mathrm{s}, \mathrm{NH}), 9.49(1 \mathrm{H}, \mathrm{s}, \mathrm{CHO}) ; \delta_{\mathrm{C}}\left(100 \mathrm{MHz} ; \mathrm{CDCl}_{3}\right) 19.5\left(\mathrm{CH}_{2}\right), 20.0\left(\mathrm{CH}_{2}\right), 29.6\left(\mathrm{CH}_{2}\right)$, $32.0\left(\mathrm{CH}_{2}\right), 52.2(\mathrm{CH}), 56.8(\mathrm{CH}), 106.4(\mathrm{C}), 111.1(\mathrm{CH}), 118.3(\mathrm{CH}), 120.0(\mathrm{CH}), 122.6(\mathrm{CH}), 126.5$ (C), $132.7(C), 136.5$ (C), $170.4(\mathrm{NC}=\mathrm{O}), 199.3$ (CHO); MS (EI) m/z 268 [M+, 75.9\%] (Found: $\mathrm{M}^{+}$, 268.12130. $\mathrm{C}_{16} \mathrm{H}_{16} \mathrm{~N}_{2} \mathrm{O}_{2}$ requires 268.12118).

(6R,12bS)-tert-butyl-6-formyl-4-oxo-1,2,3,4,6,7-hexahydro-4-oxoindolo[2,3-a]quinolizine12(12bH)-carboxylate 
$(6 R, 12 \mathrm{~b} S)-1,2,3,4,6,7,12,12 \mathrm{~b}$-octahydro-4-oxoindolo[2,3-a]quinolizine-6-carbaldehyde $\quad(3.5 \mathrm{~g}, 13.1$ $\mathrm{mmol})$ was dissolved in anhydrous tetrahydrofuran $(40.0 \mathrm{ml})$ under nitrogen. Triethylamine $(3.7 \mathrm{ml}$, $26.2 \mathrm{mmol}), N, N$-Dimethylaminopyridine $(0.35 \mathrm{~g}, 2.7 \mathrm{mmol})$ and Di-tert-butyl dicarbonate $(3.8 \mathrm{~g}, 17.1$ mmol) were added successively and the resulting solution was stirred at room temperature under nitrogen for 4 hours. After this time the volatiles were removed by rotary evaporation and the resulting residue was re-dissolved in ethyl acetate $(200 \mathrm{ml})$ and washed successively with saturated aqueous ammonium chloride $(2 \times 200 \mathrm{ml})$, saturated aqueous sodium bicarbonate $(2 \times 200 \mathrm{ml})$ and brine $(200$ $\mathrm{ml})$. The organic layer was dried over anhydrous magnesium sulphate, the solvent removed under rotary evaporation and the crude product was adsorbed onto silica and purified by flash column chromatography over silica using 3:2 ethyl acetate:hexane as eluent. The product was isolated as a yellow oil $(2.6 \mathrm{~g}, 63 \%) ;[\alpha]_{\mathrm{D}}=-925.3\left(c=3.0, \mathrm{CH}_{2} \mathrm{Cl}_{2}\right) ; v_{\max }\left(\right.$ thin film, DCM)/ $\mathrm{cm}^{-1} 1729(\mathrm{CHO}), 1653$ $(\mathrm{NBoc}[\mathrm{C}=\mathrm{O}]) ; \delta_{\mathrm{H}}\left(400 \mathrm{MHz} ; \mathrm{CDCl}_{3}\right) 1.41-1.50(1 \mathrm{H}, \mathrm{m}, \mathrm{C}=\mathrm{CCHCH}(\mathrm{H})), 1.67\left(9 \mathrm{H}, \mathrm{s}, \mathrm{OC}\left(\mathrm{CH}_{3}\right)_{3}\right)$, 1.96-2.04 (2H, m, $\left.\mathrm{CH}_{2} \mathrm{CH}_{2} \mathrm{C}=\mathrm{O}\right), 2.50-2.59(1 \mathrm{H}, \mathrm{m}, \mathrm{CH}(\mathrm{H}) \mathrm{C}=\mathrm{O}), 2.61-2.65(1 \mathrm{H}, \mathrm{m}, \mathrm{C}=\mathrm{CCHCH}(\mathrm{H}))$, 2.71-2.78 (1H, m, CH(H)C=O), $2.96(1 \mathrm{H}, \mathrm{ddd}, J 16,8,4, \mathrm{C}=\mathrm{CC} H(\mathrm{H})), 3.35-3.40(1 \mathrm{H}, \mathrm{m}$, $\mathrm{C}=\mathrm{CC} H(\mathrm{H})), 5.26-5.29(1 \mathrm{H}, \mathrm{m}, \mathrm{C}=\mathrm{CC} H), 5.92-5.94(1 \mathrm{H}, \mathrm{m}, \mathrm{NCHCHO}), 7.24-7.34(2 \mathrm{H}, \mathrm{m}, \mathrm{Ar} H)$, 7.47-7.49 (1H, m, $\operatorname{Ar} H), 8.04-8.06(1 \mathrm{H}, \mathrm{m}, \operatorname{Ar} H), 9.52(1 \mathrm{H}, \mathrm{s}, \mathrm{CHO}) ; \delta_{\mathrm{C}}\left(100 \mathrm{MHz} ; \mathrm{CDCl}_{3}\right) 19.3$ $\left(\mathrm{CH}_{2}\right), 20.5\left(\mathrm{CH}_{2}\right), 28.4\left(3 \times \mathrm{CH}_{3}\right), 30.6\left(\mathrm{CH}_{2}\right), 31.7\left(\mathrm{CH}_{2}\right), 54.0(\mathrm{CH}), 56.1(\mathrm{CH}), 84.6(\mathrm{C}), 114.7(\mathrm{C})$, $115.7(\mathrm{CH}), 118.2(\mathrm{CH}), 123.1(\mathrm{CH}), 125.0(\mathrm{CH}), 128.1(C), 134.5(C), 136.9(C), 149.8\left(\mathrm{NC}(\mathrm{O}) \mathrm{O}^{t} \mathrm{Bu}\right)$,

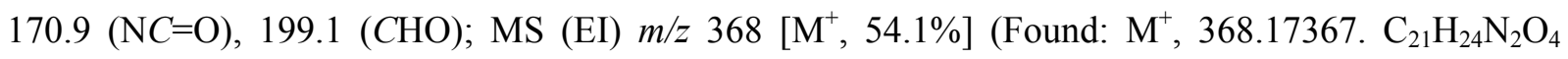
requires 368.17361$)$.

(6R,12bS)-12-(tert-butoxycarbonyl)-1,2,3,4,6,7,12,12b-octahydro-4-oxoindolo[2,3-a]quinolizine-6carboxylic acid, 8

A solution of $(6 R, 12 \mathrm{~b} S)$-tert-butyl-6-formyl-4-oxo-1,2,3,4,6,7-hexahydro-4-oxoindolo[2,3a]quinolizine-12(12bH)-carboxylate $(2.6 \mathrm{~g}, 7.1 \mathrm{mmol})$ in acetonitrile $(35.0 \mathrm{ml})$, tert-butyl alcohol $(130.0 \mathrm{ml})$ and 1-methyl-1-cyclohexene $(63.0 \mathrm{ml})$ was stirred rapidly as it was cooled to $0{ }^{\circ} \mathrm{C}$. A solution of sodium chlorite ( $80 \%$ grade $)(6.3 \mathrm{~g}, 54.7 \mathrm{mmol})$ and sodium dihydrogen phosphate $(6.3 \mathrm{~g}$, $49.7 \mathrm{mmol})$ in water $(130.0 \mathrm{ml})$ was added dropwise over a period of 10 minutes at $0{ }^{\circ} \mathrm{C}$. The solution was then allowed to stir at room temperature for a further 18 hours. After this time the reaction was partitioned between ethyl acetate $(300 \mathrm{ml})$ and brine $(200 \mathrm{ml})$. The ethyl acetate layer was then washed with $1 \mathrm{M}$ aqueous sodium dithionite solution $(100 \mathrm{ml})$. The organic layer was dried over anhydrous 
magnesium sulphate and evaporated to dryness to give the target compound which was purified by flash column chromatography over silica eluting with ethyl acetate yielding a yellow oil (1.9 g, 70\%); $[\alpha]_{\mathrm{D}}=-530\left(c=1.0, \mathrm{CH}_{2} \mathrm{Cl}_{2}\right) ; v_{\max }($ thin film, DCM $) / \mathrm{cm}^{-1} 3379(\mathrm{OH}), 1640(\mathrm{NC}=\mathrm{O}) ; \delta_{\mathrm{H}}(400 \mathrm{MHz}$; $\left.\mathrm{CDCl}_{3}\right)$ 1.34-1.40 (1H, m, C=CCHCH(H)), $1.64\left(9 \mathrm{H}, \mathrm{s}, \mathrm{OC}\left(\mathrm{CH}_{3}\right)_{3}\right), 1.82-1.91\left(2 \mathrm{H}, \mathrm{m}, \mathrm{CH}_{2} \mathrm{CH}_{2} \mathrm{C}=\mathrm{O}\right)$, 2.45-2.62 (1H, m, CH(H)C=O), 2.45-2.62 (1H, m, C=CCHCH(H)), 2.45-2.62 (1H, m, CH(H)C=O), 2.83-2.89 (1H, m, C=CCH(H)), 3.35-3.41 (1H, m, C=CCH(H)), 5.33-5.36 (1H, m, C=CCH), 5.98$6.0\left(1 \mathrm{H}, \mathrm{m}, \mathrm{NCHCO}_{2} \mathrm{H}\right), 7.18-7.28(2 \mathrm{H}, \mathrm{m}, \mathrm{Ar} H), 7.39-7.41(1 \mathrm{H}, \mathrm{m}, \operatorname{Ar} H), 8.03(1 \mathrm{H}, \mathrm{d}, J 8, \operatorname{Ar} H)$, $8.80\left(1 \mathrm{H}\right.$, br.s, $\left.\mathrm{CO}_{2} \mathrm{H}\right) ; \delta_{\mathrm{C}}\left(100 \mathrm{MHz} ; \mathrm{CDCl}_{3}\right) 19.0\left(\mathrm{CH}_{2}\right), 23.2\left(\mathrm{CH}_{2}\right), 28.2\left(3 \times \mathrm{CH}_{3}\right), 30.4\left(\mathrm{CH}_{2}\right), 31.3$ $\left(\mathrm{CH}_{2}\right), 49.8(\mathrm{CH}), 54.1(\mathrm{CH}), 84.4(\mathrm{C}), 115.0(\mathrm{C}), 115.7(\mathrm{CH}), 118.4(\mathrm{CH}), 123.0(\mathrm{CH}), 124.8(\mathrm{CH})$, $128.4(C), 133.9(C), 136.8(C), 149.9\left(\mathrm{NC}(\mathrm{O}) \mathrm{O}^{t} \mathrm{Bu}\right), 172.1(\mathrm{NC}=\mathrm{O}), 173.0(C(\mathrm{O}) \mathrm{OH}) ; \mathrm{MS}(\mathrm{EI}) \mathrm{m} / \mathrm{z}$ $384\left[\mathrm{M}^{+}, 2.0 \%\right]$ (Found: $\mathrm{M}^{+}, 384.16768 . \mathrm{C}_{21} \mathrm{H}_{24} \mathrm{~N}_{2} \mathrm{O}_{5}$ requires 384.16852).

\section{Radical Induced Decarboxylation Strategy:}

(6R,12bS)-tert-butyl-6-([phenylselanyl]carbonyl)-1,2,3,4,6,7-hexahydro-4-oxoindolo[2,3-

\section{a]quinolizine-12(12bH)-carboxylate}

To a flask containing compound $8(1.9 \mathrm{~g}, 4.95 \mathrm{mmol})$ under nitrogen was added anhydrous dichloromethane $(30.0 \mathrm{ml})$ followed by diphenyldiselenide $(2.4 \mathrm{~g}$, $7.5 \mathrm{mmol})$. The resulting mixture was cooled to $0{ }^{\circ} \mathrm{C}$ and tributylphosphine ( $90 \%$ grade) $(3.1 \mathrm{ml}, 10.1 \mathrm{mmol})$ was added dropwise. The solution was allowed to warm to room temperature and stirring continued for a further 18 hours at room temperature. Dichloromethane $(100 \mathrm{ml})$ and water $(100 \mathrm{ml})$ were added and the aqueous layer was extracted with a further $(100 \mathrm{ml})$ of dichloromethane. The combined organic fractions were washed with brine $(100 \mathrm{ml})$, dried over anhydrous magnesium sulphate and evaporated to dryness to give the crude product which was purified by flash column chromatography over silica eluting with 8:2 hexane:ethyl acetate, then 3:2 ethyl acetate:hexane giving the title compound as a pale yellow foam $(1.72 \mathrm{~g}, 66 \%) ; \mathrm{Mp} 75-77^{\circ} \mathrm{C} ;[\alpha]_{\mathrm{D}}=-84\left(c=1.0, \mathrm{CH}_{2} \mathrm{Cl}_{2}\right) ; v_{\max }($ thin film, DCM $) / \mathrm{cm}^{-1} 1729(\mathrm{COSePh})$, $1654(\mathrm{NC}=\mathrm{O}) ; \delta_{\mathrm{H}}\left(400 \mathrm{MHz} ; \mathrm{CDCl}_{3}\right) 1.44-1.54(1 \mathrm{H}, \mathrm{m}, \mathrm{C}=\mathrm{CCHCH}(\mathrm{H})), 1.70\left(9 \mathrm{H}, \mathrm{s}, \mathrm{OC}\left(\mathrm{CH}_{3}\right)_{3}\right)$, 1.95-2.11 (2H, m, $\left.\mathrm{CH}_{2} \mathrm{CH}_{2} \mathrm{C}=\mathrm{O}\right), 2.60-2.68(1 \mathrm{H}, \mathrm{m}, \mathrm{C}=\mathrm{CCHCH}(\mathrm{H})), 2.60-2.68(1 \mathrm{H}, \mathrm{m}, \mathrm{CH}(\mathrm{H}) \mathrm{C}=\mathrm{O})$, 2.78-2.85 (1H, m, $\mathrm{C} H(\mathrm{H}) \mathrm{C}=\mathrm{O}), 2.87-2.93(1 \mathrm{H}, \mathrm{m}, \mathrm{C}=\mathrm{CC} H(\mathrm{H})), 3.47-3.52(1 \mathrm{H}, \mathrm{m}, \mathrm{C}=\mathrm{CC} H(\mathrm{H}))$, 5.56-5.59 (1H, m, NCHCOSePh), 6.15-6.17 (1H, m, ArH), 7.24-7.32 (5H, m, ArH), 7.39-7.40 (2H, m, ArH), 7.43-7.46 (1H, m, ArH), 8.03-8.06 (1H, m, ArH); $\delta_{\mathrm{C}}\left(100 \mathrm{MHz} ; \mathrm{CDCl}_{3}\right) 19.0\left(\mathrm{CH}_{2}\right), 22.4$ $\left(\mathrm{CH}_{2}\right), 28.3\left(3 \times \mathrm{CH}_{3}\right), 31.1\left(\mathrm{CH}_{2}\right), 31.8\left(\mathrm{CH}_{2}\right), 54.1(\mathrm{CH}), 59.6(\mathrm{CH}), 84.5(\mathrm{C}), 114.8(\mathrm{C}), 115.7(\mathrm{CH})$, 
$118.4(\mathrm{CH}), 123.1(\mathrm{CH}), 124.9(\mathrm{CH}), 125.4(C), 128.3(\mathrm{C}), 128.9(\mathrm{CH}), 129.2(2 \times C \mathrm{H}), 133.5(C)$, $136.0(2 \times C \mathrm{H}), 136.8(C), 149.9\left(\mathrm{NC}(\mathrm{O}) \mathrm{O}^{t} \mathrm{Bu}\right), 171.6(\mathrm{NC}=\mathrm{O}), 200.3(\mathrm{COSePh}) ; \mathrm{MS}(\mathrm{CI}) \mathrm{m} / z 525$ $\left[\mathrm{MH}^{+}, 6.7 \%\right]$ (Found: $\mathrm{MH}^{+}$, 525.13013. $\mathrm{C}_{27} \mathrm{H}_{29} \mathrm{~N}_{2} \mathrm{O}_{4}$ Se requires 525.12925).

(12bS)-tert-butyl-1,2,3,4,6,7-hexahydro-4-oxoindolo[2,3-a]quinolizine-12(12bH)-carboxylate, 9

A three-necked flask fitted with a condenser, glass stopper and a suba seal was flushed with nitrogen. A solution of (6R,12bS)-tert-butyl-6-([phenylselanyl]carbonyl)-1,2,3,4,6,7-hexahydro-4-oxoindolo[2,3a]quinolizine-12(12bH)-carboxylate $(1.7 \mathrm{~g}, 3.2 \mathrm{mmol})$ in anhydrous toluene $(20.0 \mathrm{ml})$ was added via cannula. The solution was then degassed with nitrogen for 15 minutes before adding tri- $n$-butyltin hydride ( $3.5 \mathrm{ml}, 13.2 \mathrm{mmol})$ via syringe. The resulting mixture was heated to $80{ }^{\circ} \mathrm{C}$ in an oil bath whereupon azobisisobutyronitrile $(0.1 \mathrm{~g}, 0.6 \mathrm{mmol})$ was added portionwise over a 2 hour period at 80 ${ }^{\circ} \mathrm{C}$. After 2 hours stirring at $80{ }^{\circ} \mathrm{C}$ the mixture was cooled to room temperature and the solvent removed on the rotary evaporator. The resulting crude oil was adsorbed onto silica and purified by flash column chromatography over silica eluting with hexane followed by 3:1 ethyl acetate:hexane. The target compound was isolated as a colourless oil $(1.1 \mathrm{~g}, 98 \%) ;[\alpha]_{\mathrm{D}}=-77.3\left(c=1.8, \mathrm{CH}_{2} \mathrm{Cl}_{2}\right)$; $v_{\max }($ thin film, DCM $) / \mathrm{cm}^{-1} 1732\left(\mathrm{NC}=\mathrm{O}(\mathrm{O})^{t} \mathrm{Bu}\right), 1644(\mathrm{NC}=\mathrm{O}) ; \delta_{\mathrm{H}}\left(400 \mathrm{MHz} ; \mathrm{CDCl}_{3}\right) 1.32-1.42(1 \mathrm{H}$, $\mathrm{m}, \mathrm{C}=\mathrm{CCHCH}(\mathrm{H})), 1.67\left(9 \mathrm{H}, \mathrm{s}, \mathrm{OC}\left(\mathrm{CH}_{3}\right)_{3}\right), 1.79-1.86\left(2 \mathrm{H}, \mathrm{m}, \mathrm{CH}_{2} \mathrm{CH}_{2} \mathrm{C}=\mathrm{O}\right), 2.34-2.43(1 \mathrm{H}, \mathrm{m}$, $\mathrm{C} H(\mathrm{H}) \mathrm{C}=\mathrm{O}), 2.54-2.77(1 \mathrm{H}, \mathrm{m}, \mathrm{C}=\mathrm{CCHCH}(\mathrm{H})), 2.54-2.77(1 \mathrm{H}, \mathrm{m}, \mathrm{CH}(\mathrm{H}) \mathrm{C}=\mathrm{O}), 2.54-2.77(2 \mathrm{H}, \mathrm{m}$, $\left.\mathrm{CH}_{2} \mathrm{CH}_{2} \mathrm{~N}\right), 2.54-2.77(1 \mathrm{H}, \mathrm{m}, \mathrm{CH}(\mathrm{H}) \mathrm{N}), 5.05-5.15(1 \mathrm{H}, \mathrm{m}, \mathrm{CH}(\mathrm{H}) \mathrm{N}), 5.05-5.15(1 \mathrm{H}, \mathrm{m}, \mathrm{C}=\mathrm{CCH})$, 7.18-7.30 (2H, m, ArH), $7.37(1 \mathrm{H}, \mathrm{d}, J$ 8, $\mathrm{ArH}), 8.06(1 \mathrm{H}, \mathrm{d}, J$ 8, $\mathrm{Ar} H) ; \delta_{\mathrm{C}}\left(100 \mathrm{MHz} ; \mathrm{CDCl}_{3}\right) 19.5$ $\left(\mathrm{CH}_{2}\right), 21.6\left(\mathrm{CH}_{2}\right), 28.1\left(3 \times \mathrm{CH}_{3}\right), 30.2\left(\mathrm{CH}_{2}\right), 32.1\left(\mathrm{CH}_{2}\right), 38.8\left(\mathrm{CH}_{2}\right), 56.0(\mathrm{CH}), 84.1(\mathrm{C}), 115.5$ $(\mathrm{CH}), 118.2(\mathrm{CH}), 118.2(\mathrm{C}), 122.9(\mathrm{CH}), 124.5(\mathrm{CH}), 128.6(C), 135.2(C), 136.8(C), 150.1$

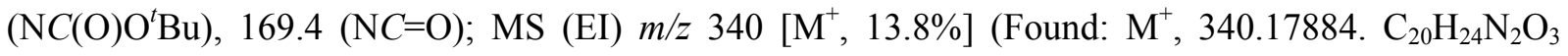
requires 340.17862 ).

\section{(12bS)-1,2,3,6,7,12b-hexahydroindolo[2,3-a]quinolizin-4(12H)-one, 10}

Compound 9 (0.23 g, $0.68 \mathrm{mmol})$ was dissolved in anhydrous tetrahydrofuran $(10.0 \mathrm{ml})$ under a nitrogen atmosphere. A 1.0 M solution of tetrabutylammonium fluoride in tetrahydrofuran $(6.8 \mathrm{ml}, 6.8$ mmol) was then added and the mixture was heated under reflux for 9 hours. After this time the mixture was cooled to room temperature and water $(40.0 \mathrm{ml})$ was added. The mixture was extracted with ethyl 
acetate $(2 \times 70.0 \mathrm{ml})$ and the organic fractions were dried over anhydrous magnesium sulphate and the solvent removed on the rotary evaporator. The crude product was adsorbed onto silica and purified by flash column chromatography over silica using ethyl acetate as eluent to give the target compound as an off-white solid $(0.1 \mathrm{~g}, 63 \%), \mathrm{Mp} 238-240{ }^{\circ} \mathrm{C} ;[\alpha]_{\mathrm{D}}=-286\left(c=1.0, \mathrm{CHCl}_{3}\right) ; v_{\max }$ (thin film, $\mathrm{DCM}) / \mathrm{cm}^{-1} 1651(\mathrm{NC}=\mathrm{O}) ; \delta_{\mathrm{H}}\left(400 \mathrm{MHz} ; \mathrm{CDCl}_{3}\right) 1.76-1.89\left(1 \mathrm{H}, \mathrm{m}, \mathrm{CH}(\mathrm{H}) \mathrm{CH}_{2} \mathrm{C}=\mathrm{O}\right), 1.76-1.89(1 \mathrm{H}$, $\left.\mathrm{m}, \quad \mathrm{C} H(\mathrm{H}) \mathrm{CH}_{2} \mathrm{CH}_{2} \mathrm{C}=\mathrm{O}\right), \quad 1.95-2.01 \quad\left(1 \mathrm{H}, \quad \mathrm{m}, \quad \mathrm{CH}(\mathrm{H}) \mathrm{CH}_{2} \mathrm{C}=\mathrm{O}\right), \quad 2.36-2.47 \quad(1 \mathrm{H}, \quad \mathrm{m}$, $\left.\mathrm{C} H(\mathrm{H}) \mathrm{CH}_{2} \mathrm{CH}_{2} \mathrm{C}=\mathrm{O}\right), 2.36-2.47(1 \mathrm{H}, \mathrm{m}, \mathrm{CH}(\mathrm{H}) \mathrm{C}=\mathrm{O}), 2.57-2.62(1 \mathrm{H}, \mathrm{m}, \mathrm{CH}(\mathrm{H}) \mathrm{C}=\mathrm{O}), 2.72-2.82$ $\left(1 \mathrm{H}, \mathrm{m}, \mathrm{CH}(\mathrm{H}) \mathrm{CH}_{2} \mathrm{~N}\right), 2.84-2.89\left(1 \mathrm{H}, \mathrm{m}, \mathrm{CH}(\mathrm{H}) \mathrm{CH}_{2} \mathrm{~N}\right), 2.84-2.89(1 \mathrm{H}, \mathrm{m}, \mathrm{CH}(\mathrm{H}) \mathrm{N}), 4.76-4.80(1 \mathrm{H}$, $\mathrm{m}, \mathrm{C}=\mathrm{CC} H), 5.14-5.22(1 \mathrm{H}, \mathrm{m}, \mathrm{CH}(\mathrm{H}) \mathrm{N}), 7.11-7.21(2 \mathrm{H}, \mathrm{m}, \mathrm{ArH}), 7.33-7.36(1 \mathrm{H}, \mathrm{m}, \mathrm{ArH}), 7.51$ $(1 \mathrm{H}, \mathrm{d}, J$ 8, $\mathrm{ArH}), 7.85(1 \mathrm{H}, \mathrm{br}, \mathrm{s}, \mathrm{NH}) ; \delta_{\mathrm{C}}\left(100 \mathrm{MHz} ; \mathrm{CDCl}_{3}\right) 19.4\left(\mathrm{CH}_{2}\right), 21.0\left(\mathrm{CH}_{2}\right), 29.1\left(\mathrm{CH}_{2}\right), 32.5$ $\left(\mathrm{CH}_{2}\right), 40.1\left(\mathrm{CH}_{2}\right), 54.4(\mathrm{CH}), 109.8(\mathrm{C}), 110.9(\mathrm{CH}), 118.5(\mathrm{CH}), 119.9(\mathrm{CH}), 122.2(\mathrm{CH}), 127.0(\mathrm{C})$, $133.3(C), 136.2(C), 169.1(\mathrm{NC}=\mathrm{O})$; $\mathrm{MS}(\mathrm{EI}) \mathrm{m} / z 240\left[\mathrm{M}^{+}, 100.0 \%\right]$ (Found: $\mathrm{M}^{+}, 240.12632$. $\mathrm{C}_{15} \mathrm{H}_{16} \mathrm{~N}_{2} \mathrm{O}$ requires 240.12626).

\section{(12bS)-1,2,3,4,6,7,12,12b-octahydroindolo[2,3-a]quinolizine, 11}

Lithium aluminium hydride $(0.07 \mathrm{~g}, 1.68 \mathrm{mmol})$ was weighed into a pre-dried three necked flask fitted with a condenser under a nitrogen atmosphere. Anhydrous tetrahydrofuran $(13.0 \mathrm{ml})$ was added and the suspension was cooled to $0{ }^{\circ} \mathrm{C}$ with an ice bath. Compound $10(0.07 \mathrm{~g}, 1.68 \mathrm{mmol})$ in anhydrous tetrahydrofuran $(4.0 \mathrm{ml})$ was added dropwise to the hydride solution at $0{ }^{\circ} \mathrm{C}$. The resulting mixture was heated under reflux for 3 hours and then stirred at room temperature for a further 12 hours. Ether (5.0 $\mathrm{ml}$ ) was added and the reaction was quenched by the careful addition of saturated aqueous sodium potassium tartrate solution. The mixture was stirred for another 1 hour before the addition of anhydrous magnesium sulphate prior to filtration through a celite pad. The reaction vessel was washed out with ethyl acetate and filtered through the celite pad. The filtrate was evaporated under reduced pressure to yield the crude product which was adsorbed onto silica and purified by flash column chromatography over silica using ethyl acetate as eluent to give the target compound as a yellow solid ( $0.09 \mathrm{~g}, 96 \%)$, a small portion of which was recrystallised from dichloromethane to yield yellow plate-like crystals; $\mathrm{Mp}$ $146-148{ }^{\circ} \mathrm{C}\left(\right.$ lit. $\left.{ }^{[13 \mathrm{a}]} \mathrm{Mp} 149-150{ }^{\circ} \mathrm{C}\right) ;[\alpha]_{\mathrm{D}}=-79.6(c=1.0, \mathrm{MeOH})\left(\right.$ lit. ${ }^{[13 \mathrm{a}]}[\alpha]_{\mathrm{D}}=-86.5 \pm 2(c=1.0$, $\mathrm{MeOH})$ ); $v_{\max }\left(\right.$ thin film, DCM) $/ \mathrm{cm}^{-1} 3441(\mathrm{NH}) ; \delta_{\mathrm{H}}\left(400 \mathrm{MHz} ; \mathrm{CDCl}_{3}\right)$ 1.40-1.52 (1H, m, $\left.\mathrm{C}=\mathrm{CCHCH}_{2} \mathrm{CH}(\mathrm{H})\right), \quad 1.57 \quad(1 \mathrm{H}, \quad \mathrm{ddd}, \quad J \quad 24, \quad 16, \quad 4, \mathrm{C}=\mathrm{CCHCH}(\mathrm{H})), \quad 1.69-1.77 \quad(2 \mathrm{H}, \mathrm{m}$, $\left.\mathrm{C}=\mathrm{CCH}\left(\mathrm{CH}_{2}\right)_{2} \mathrm{CH}_{2}\right), 1.85-1.89\left(1 \mathrm{H}, \mathrm{m}, \mathrm{C}=\mathrm{CCHCH}_{2} \mathrm{CH}(\mathrm{H})\right), 2.02-2.05(1 \mathrm{H}, \mathrm{m}, \mathrm{C}=\mathrm{CCHCH}(\mathrm{H})), 2.37$ 
$\left(1 \mathrm{H}, \mathrm{dt}, J\right.$ 8, 4, $\left.\mathrm{C}=\mathrm{CCH}\left(\mathrm{CH}_{2}\right)_{3} \mathrm{CH}(\mathrm{H}) \mathrm{N}\right), 2.58-2.73\left(1 \mathrm{H}, \mathrm{m}, \mathrm{C}=\mathrm{CCH}_{2} \mathrm{CH}(\mathrm{H}) \mathrm{N}\right), 2.58-2.73(1 \mathrm{H}, \mathrm{m}$, $\left.\mathrm{C}=\mathrm{CC} H(\mathrm{H}) \mathrm{CH}_{2} \mathrm{~N}\right), 2.96-3.02\left(1 \mathrm{H}, \mathrm{m}, \mathrm{C}=\mathrm{CCH}_{2} \mathrm{CH}(\mathrm{H}) \mathrm{N}\right), 2.96-3.02\left(1 \mathrm{H}, \mathrm{m}, \mathrm{C}=\mathrm{CCH}(\mathrm{H}) \mathrm{CH}_{2} \mathrm{~N}\right)$, 3.02-3.08 (1H, m, C=CCH(CH$\left.)_{3} \mathrm{CH}(\mathrm{H}) \mathrm{N}\right), 3.21(1 \mathrm{H}, \mathrm{d}, J$ 8, C=CCH), 7.06-7.15 (2H, m, ArH), 7.28 $(1 \mathrm{H}, \mathrm{d}, J 8, \mathrm{Ar} H), 7.46(1 \mathrm{H}, \mathrm{d}, J$ 8, $\mathrm{ArH}), 7.73(1 \mathrm{H}, \mathrm{br}, \mathrm{s}, \mathrm{NH}) ; \delta_{\mathrm{C}}\left(100 \mathrm{MHz} ; \mathrm{CDCl}_{3}\right) 21.6\left(\mathrm{CH}_{2}\right), 24.3$ $\left(\mathrm{CH}_{2}\right), 25.7\left(\mathrm{CH}_{2}\right), 30.0\left(\mathrm{CH}_{2}\right), 53.6\left(\mathrm{CH}_{2}\right), 55.7\left(\mathrm{CH}_{2}\right), 60.2(\mathrm{CH}), 108.2(\mathrm{C}), 110.7(\mathrm{CH}), 118.1(\mathrm{CH})$, $119.4(\mathrm{CH}), 121.3(\mathrm{CH}), 127.5(C), 135.1(C), 136.0(C)$; MS (EI) $m / z 226$ [M $\mathrm{M}^{+}, 72.3 \%$ ] (Found: $\mathrm{M}^{+}$, 226.14700. $\mathrm{C}_{15} \mathrm{H}_{18} \mathrm{~N}_{2}$ requires 226.14700).

(12bR)-(E)-tert-butyl-3-ethylidene-1,2,3,4,6,7-hexahydro-4-oxoindolo[2,3-a]quinolizine-12(12b)carboxylate, 12

To a stirred solution of diisopropylamine $(1.3 \mathrm{ml}, 8.8 \mathrm{mmol})$ in anhydrous tetrahydrofuran $(10.0 \mathrm{ml})$ was added $n$-butyllithium $(2.5 \mathrm{M}$ solution in hexanes) $(3.5 \mathrm{ml}, 8.8 \mathrm{mmol})$ dropwise at $0{ }^{\circ} \mathrm{C}$ under nitrogen. The reaction mixture was stirred for 15 minutes at $0{ }^{\circ} \mathrm{C}$ and then cooled to $-78{ }^{\circ} \mathrm{C}$ whereupon a solution of (12bR)-tert-butyl-1,2,3,4,6,7-hexahydro-4oxoindolo[2,3-a]quinolizine-12(12b)-carboxylate $\quad \boldsymbol{R}-9 \quad(1.0 \mathrm{~g}, 2.9 \mathrm{mmol})$ in anhydrous tetrahydrofuran $(15.0 \mathrm{ml})$ was added via cannula. The resulting mixture was stirred for a further 30 minutes at $-78{ }^{\circ} \mathrm{C}$ after which time acetaldehyde $(1.6 \mathrm{ml}, 29.0 \mathrm{mmol})$ was added dropwise via syringe. The reaction was allowed to warm to room temperature overnight and then quenched by the addition of saturated aqueous ammonium chloride solution $(30.0 \mathrm{ml})$ and extracted with ethyl acetate $(2 \times 50 \mathrm{ml})$. The combined organic extracts were dried over anhydrous magnesium sulphate and the solvent was removed under reduced pressure to yield a yellow oil. The crude oil was dissolved in anhydrous dichloromethane $(15.0 \mathrm{ml})$ under a nitrogen atmosphere. Triethylamine $(1.2 \mathrm{ml}, 8.7 \mathrm{mmol})$ was added and the mixture cooled to $40{ }^{\circ} \mathrm{C}$ before adding methane sulfonyl chloride $(0.34 \mathrm{ml}, 4.4 \mathrm{mmol})$. After 20 minutes stirring at $-40{ }^{\circ} \mathrm{C}$ the reaction was allowed to warm to room temperature and stirred for an additional 3 hours. After this time the solvent was removed and the mesylate dissolved in anhydrous tetrahydrofuran $(35.0 \mathrm{ml})$ to which 1,5-diazabicyclo[4.3.0]non-5-ene $(1.1 \mathrm{ml}, 8.7 \mathrm{mmol})$ was added and stirred at room temperature for 16 hours. The solvent was removed and the residue re-dissolved in dichloromethane $(80 \mathrm{ml})$ which was washed with saturated aqueous ammonium chloride solution $(60 \mathrm{ml})$. The organic fraction was dried over anhydrous 
magnesium sulphate and evaporated to dryness to give the crude product. $250 \mathrm{MHz}{ }^{1} \mathrm{H} \mathrm{NMR}$ spectroscopy of the crude product revealed formation of the product as a single trans geometrical isomer which was purified by flash column chromatography over silica using $5: 1$ hexane:ethyl acetate as eluent $(0.70 \mathrm{~g}, 65 \%)$.

Trans isomer 12 isolated as a pale yellow oil; $[\alpha]_{\mathrm{D}}=+140.4\left(c=2.0, \mathrm{CH}_{2} \mathrm{Cl}_{2}\right) ; v_{\max }($ thin film, $\mathrm{DCM}) / \mathrm{cm}^{-1} 1734\left(\mathrm{NC}=\mathrm{O}(\mathrm{O})^{t} \mathrm{Bu}\right) 1591(\mathrm{C}=\mathrm{C}) ; \delta_{\mathrm{H}}\left(400 \mathrm{MHz} ; \mathrm{CDCl}_{3}\right)$ 1.47-1.52 (1H, m), 1.70 $(9 \mathrm{H}, \mathrm{s}), 1.77(3 \mathrm{H}, \mathrm{d}, J$ 8), 2.40-2.49 (1H, m), 2.58-2.64 (1H, m), 2.71-2.72 (1H, m), 2.73$2.76(2 \mathrm{H}, \mathrm{m}), 2.84-2.90(1 \mathrm{H}, \mathrm{m}), 5.13-5.23(2 \mathrm{H}, \mathrm{m}), 7.00-7.05(1 \mathrm{H}, \mathrm{m}), 7.23-7.32(2 \mathrm{H}, \mathrm{m})$, 7.43-7.46 (1H, m), 8.06-8.08 (1H, m); $\delta_{\mathrm{C}}\left(100 \mathrm{MHz} ; \mathrm{CDCl}_{3}\right) 13.7\left(\mathrm{CH}_{3}\right), 21.5\left(\mathrm{CH}_{2}\right), 23.4$ $\left(\mathrm{CH}_{2}\right), 28.2\left(3 \times \mathrm{XH}_{3}\right), 30.3\left(\mathrm{CH}_{2}\right), 39.1\left(\mathrm{CH}_{2}\right), 55.1(\mathrm{CH}), 84.2(\mathrm{C}), 115.6(\mathrm{CH}), 118.2(C)$, $118.3(\mathrm{CH}), 123.0(\mathrm{CH}), 124.5(\mathrm{CH}), 128.6(C), 129.3(C), 134.2(\mathrm{CH}), 135.0(C), 136.7(C)$, $150.2\left(\mathrm{NC}(\mathrm{O}) \mathrm{O}^{t} \mathrm{Bu}\right), 164.9(\mathrm{NC}=\mathrm{O})$; MS (EI) $m / z 366$ [M+, 8.9\%] (Found: $\mathrm{M}^{+}, 366.19484$. $\mathrm{C}_{22} \mathrm{H}_{26} \mathrm{~N}_{2} \mathrm{O}_{3}$ requires 366.19434).

When the DBN elimination was carried out in a 1:1 mixture of tetrahydrofuran and dichloromethane a 4.7:1 mixture of trans:cis geometrical isomers was obtained; these were separated by column chromatography over silica using 5:1 hexane:ethyl acetate as eluent.

Cis isomer isolated as a yellow oil; $[\alpha]_{\mathrm{D}}=+218.4\left(c=4.0, \mathrm{CH}_{2} \mathrm{Cl}_{2}\right) ; v_{\max }($ thin film, $\mathrm{DCM}) / \mathrm{cm}^{-1} 1731\left(\mathrm{NC}=\mathrm{O}(\mathrm{O})^{t} \mathrm{Bu}\right) 1660(\mathrm{C}=\mathrm{C}) ; \delta_{\mathrm{H}}\left(400 \mathrm{MHz} ; \mathrm{CDCl}_{3}\right) 1.22-1.533(2 \mathrm{H}, \mathrm{m}), 1.69$ (9H, s), $2.17(3 \mathrm{H}, \mathrm{d}, J 8), 2.47-2.52(1 \mathrm{H}, \mathrm{m}), 2.57-2.62(1 \mathrm{H}, \mathrm{m}), 2.73-2.78(2 \mathrm{H}, \mathrm{m}), 2.80-$ $2.88(1 \mathrm{H}, \mathrm{m}), 5.06-5.18(2 \mathrm{H}, \mathrm{m}), 5.92-5.97(1 \mathrm{H}, \mathrm{m}), 7.23-7.32(2 \mathrm{H}, \mathrm{m}), 7.44-7.47(1 \mathrm{H}, \mathrm{m})$, 8.04-8.06 (1H, m); $\delta_{\mathrm{C}}\left(100 \mathrm{MHz} ; \mathrm{CDCl}_{3}\right) 15.8\left(\mathrm{CH}_{3}\right), 21.6\left(\mathrm{CH}_{2}\right), 28.2(3 \mathrm{xCH}), 30.3\left(\mathrm{CH}_{2}\right)$, $30.6\left(\mathrm{CH}_{2}\right), 38.8\left(\mathrm{CH}_{2}\right), 55.7(\mathrm{CH}), 84.2(\mathrm{C}), 115.5(\mathrm{CH}), 118.3(\mathrm{CH}), 118.4(\mathrm{C}), 123.0(\mathrm{CH})$, $\left.124.5(\mathrm{CH}), 128.7(C), 128.7(C), 135.4(\mathrm{CH}), 136.8(C), 136.8(\mathrm{CH}), 150.2(\mathrm{NC}(\mathrm{O}))^{t} \mathrm{Bu}\right)$, $165.4\left(\mathrm{NC}=\mathrm{O}\right.$ ); MS (EI) $m / z 366\left[\mathrm{M}^{+}, 13.1 \%\right.$ ] (Found: $\mathrm{M}^{+}$, 366.19395. $\mathrm{C}_{22} \mathrm{H}_{26} \mathrm{~N}_{2} \mathrm{O}_{3}$ requires 366.19434).

(12bR)-(E)-3-ethylidene-1,2,3,6,7,12b-hexahydroindolo[2,3-a]quinolizin-4(12H)-one, 13 
Compound 12 (0.7 g, $1.91 \mathrm{mmol})$ was dissolved in anhydrous tetrahydrofuran $(8.0 \mathrm{ml})$ under a nitrogen atmosphere. A $1.0 \mathrm{M}$ solution of tetrabutyl ammonium fluoride in tetrahydrofuran (19.1 ml, $19.1 \mathrm{mmol})$ was then added and the mixture was heated under reflux for 9 hours. After this time the mixture was cooled to room temperature and water $(30.0 \mathrm{ml})$ was added. The mixture was extracted with ethyl acetate $(2 \times 50.0 \mathrm{ml})$ and the organic fractions were dried over anhydrous magnesium sulphate and the solvent removed on the rotary evaporator. The crude product was adsorbed onto silica and purified by flash column chromatography over silica using 3:1 hexane:ethyl acetate as eluent to give the target compound as a yellow solid $(0.32 \mathrm{~g}, 63 \%)$, a portion of which was recrystallised from diethyl ether to give a yellow crystalline solid; $\mathrm{Mp} 198-202{ }^{\circ} \mathrm{C} ;[\alpha]_{\mathrm{D}}=+77.2\left(c=1.0, \mathrm{CH}_{2} \mathrm{Cl}_{2}\right) ; v_{\max }\left(\right.$ thin film, $\left.\mathrm{CH}_{2} \mathrm{Cl}_{2}\right) / \mathrm{cm}^{-1}$ $3238(\mathrm{NH}), 1746(\mathrm{NC}=\mathrm{O}) ; \delta_{\mathrm{H}}\left(400 \mathrm{MHz} ; \mathrm{CDCl}_{3}\right)$ 1.72-1.82 (1H, m), $1.77(3 \mathrm{H}, \mathrm{dd}, J$ 8, 4), 2.31-2.41 (1H, m), 2.50-2.58 (1H, m), 2.77-2.97 (4H, m), 4.81-4.84 (1H, m), 5.18-5.25 (1H, m), 7.00-7.06 (1H, m), 7.09-7.19 (2H, m), 7.31-7.34 (1H, m), 7.49-7.51 (1H, m, ArH) 8.57 $(1 \mathrm{H}, \mathrm{br}, \mathrm{s}, \mathrm{NH}) ; \delta_{\mathrm{C}}\left(100 \mathrm{MHz} ; \mathrm{CDCl}_{3}\right) 13.7\left(\mathrm{CH}_{3}\right), 21.1\left(\mathrm{CH}_{2}\right), 22.7\left(\mathrm{CH}_{2}\right), 28.9\left(\mathrm{CH}_{2}\right), 40.6$ $\left(\mathrm{CH}_{2}\right), 54.0(\mathrm{CH}), 109.4(\mathrm{C}), 111.0(\mathrm{CH}), 118.3(\mathrm{C}), 119.6(\mathrm{CH}), 122.0(\mathrm{CH}), 126.8(C), 129.2$ (C), $133.4(C), 134.1(C H), 136.4(C), 164.7(\mathrm{NC}=\mathrm{O})$; MS (EI) $m / z 266$ [M+1, 100\%] (Found: $\mathrm{M}^{+}$, 266.14191. $\mathrm{C}_{17} \mathrm{H}_{18} \mathrm{~N}_{2} \mathrm{O}$ requires 266.14191).

\section{(12bR)-(E)-3-ethylidene-1,2,3,4,6,7,12,12b-octahydroindolo[2,3-a]quinolizine, 14}

A slurry of compound $\mathbf{1 3}(0.32 \mathrm{~g}, 1.2 \mathrm{mmol})$ and trimethyloxonium tetrafluoroborate $(0.48 \mathrm{~g}$, $3.3 \mathrm{mmol})$ in anhydrous dichloromethane $(40.0 \mathrm{ml})$ containing 2,6-di-tert-butylpyridine $(0.80$ $\mathrm{ml}, 3.5 \mathrm{mmol}$ ) was stirred at room temperature under nitrogen for 21 hours. After this time the reaction was cooled to $0{ }^{\circ} \mathrm{C}$ and anhydrous methanol $(16.0 \mathrm{ml})$ was added. After 15 minutes sodium borohydride $(0.48 \mathrm{~g}, 12.0 \mathrm{mmol})$ was added carefully and the mixture stirred at $0{ }^{\circ} \mathrm{C}$ for a further 30 minutes. The reaction was warmed to room temperature and saturated aqueous sodium bicarbonate solution $(40.0 \mathrm{ml})$ and ethyl acetate $(40.0 \mathrm{ml})$ were added. The layers were separated and the aqueous layer extracted with ethyl acetate $(2 \times 30.0 \mathrm{ml})$. The combined organic fractions were dried over anhydrous magnesium sulphate and the solvent removed under reduced pressure to give the crude product which was adsorbed onto silica and purified 
by flash column chromatography over silica eluting with 98:2 ethyl acetate:triethylamine. The target compound was isolated as a yellow solid $(0.23 \mathrm{~g}, 77 \%)$, a portion of which was recrystallised from dichloromethane to yield yellow needles; Mp 139.5-140.5 ${ }^{\circ} \mathrm{C}$ (lit. ${ }^{[13 \mathrm{a}]} \mathrm{Mp}$ $\left.140{ }^{\circ} \mathrm{C}\right) ;[\alpha]_{\mathrm{D}}=+52.0\left(c=1.0, \mathrm{CHCl}_{3}\right)\left(\right.$ lit. $\left.^{[13 \mathrm{a}]}[\alpha]_{\mathrm{D}}=+56 \pm 2\left(c=1.0, \mathrm{CHCl}_{3}\right)\right) ; v_{\max }($ thin film, DCM) $/ \mathrm{cm}^{-1} 3455(\mathrm{NH}) ; \delta_{\mathrm{H}}\left(400 \mathrm{MHz} ; \mathrm{CDCl}_{3}\right) 1.52-1.60(1 \mathrm{H}, \mathrm{m}), 1.63(3 \mathrm{H}, \mathrm{d}, J 8)$, 1.95-2.02 (1H, m), 2.15-2.20 (1H, m), 2.61-2.75 (2H, m), 2.80-2.84 (1H, m), 2.98-3.11 (3H, m), 3.32-3.35 (1H, m), 3.38-3.41 (1H, m), $5.43(1 \mathrm{H}, \mathrm{q}, J$ 8), 7.06-7.15 (2H, m), 7.30-7.33 $(1 \mathrm{H}, \mathrm{m}), 7.46-7.48(1 \mathrm{H}, \mathrm{m}), 7.76(1 \mathrm{H}, \mathrm{br}, \mathrm{s}) ; \delta_{\mathrm{C}}\left(100 \mathrm{MHz} ; \mathrm{CDCl}_{3}\right) 12.7\left(\mathrm{CH}_{3}\right), 21.6\left(\mathrm{CH}_{2}\right)$, $25.9\left(\mathrm{CH}_{2}\right), 30.3\left(\mathrm{CH}_{2}\right), 52.9\left(\mathrm{CH}_{2}\right), 60.2(\mathrm{CH}), 63.5\left(\mathrm{CH}_{2}\right), 108.4(\mathrm{C}), 110.7(\mathrm{CH}), 118.2$ $(\mathrm{CH}), 119.4(\mathrm{CH}), 119.4(\mathrm{CH}), 121.3(\mathrm{CH}), 127.4(\mathrm{C}), 134.0(\mathrm{C}), 134.6(C), 136.0(\mathrm{C})$; MS (EI) $m / z 252\left[\mathrm{M}^{+}, 98.6 \%\right]$ (Found: $\mathrm{M}^{+}, 252.16261 . \mathrm{C}_{17} \mathrm{H}_{20} \mathrm{~N}_{2}$ requires 252.16265).

\section{X-ray Crystal Structure of $\boldsymbol{R}-14$}

(CCDC no: 261811). Crystal data for 14·1/2 $\mathrm{H}_{2} \mathrm{O}: \mathrm{C}_{17} \mathrm{H}_{21} \mathrm{~N}_{2} \mathrm{O}_{0.5}, M=261.36$, monoclinic, $P 2_{1}$, $a=12.7620(16), b=6.8946(9), c=17.222(2) \AA, \beta=98.533(2)^{\circ}, V=1498.5(3) \AA^{3}, Z=4, D_{\mathrm{c}}$ $=1.158 \mathrm{~g} \mathrm{~cm}^{-3}, \mu($ Mo-K $\mathrm{a})=0.070 \mathrm{~mm}^{-1}, T=150(2) \mathrm{K}$, yellow blocks; 8486 reflections measured on a Bruker SMART 1000 CCD diffractometer, of which 4933 were independent, data corrected for absorption on the basis of symmetry equivalent and repeated data (min and max transmission factors: $0.960,0.987)$ and Lp effects, $R_{\text {int }}=0.032$, structure solved by direct methods, $F^{2}$ refinement, $R_{1}=0.043$ for 3705 data with $F^{2}>2 \mathrm{~s}\left(F^{2}\right), w R_{2}=0.105$ for all data, 367 parameters. These data can be obtained free of charge via www.ccdc.cam.ac.uk/conts/retrieving.html (or from the CCDC, 12 Union Road, Cambridge, CB2 1EZ, UK. Fax +44 1223 336033, e-mail: deposit@,ccdc.cam.ac.uk.).

\section{References and Notes}

[1] S. F. Martin, K. X. Chen, C. T. Early, Org. Lett. 1999, 1, 79-81.

[2] J. Yu, T. Wang, X. Liu, J. Deschamps, J. Flippen-Anderson, X. Liao, J. M. Cook, J. Org. Chem. 2003, 68, 7565-7581.

[3] M. Logers, L. E. Overman, G. S. Welmaker, J. Am. Chem. Soc. 1995, 117, 9139-9150. 
[4] A. Deiters, K. Chen, C. T. Early, S. F. Martin, J. Am. Chem. Soc. 2003, 125, 45414550 .

[5] A. Deiters, S. F. Martin, Org. Lett. 2002, 4, 3243-3245.

[6] R. S. Fornicola, K. Subburaj, J. Montgomery, Org. Lett. 2002, 4, 615-617.

[7] X. Liu, T. Wang, Q. Xu, C. Ma, J. M. Cook, Tetrahedron Lett. 2000, 41, 6299-6303.

[8] a) S. M. Allin, C. I. Thomas, J. E. Allard, M. Duncton, M. R. J. Elsegood, M. Edgar, Tetrahedron Lett. 2003, 44, 2335-2337; b) S. M. Allin, D. G. Vaidya, S. L. James, J. E. Allard, T. A. D. Smith, V. McKee, W. P. Martin, Tetrahedron Lett. 2002, 43, 3661-3663; c) S. M. Allin, S. L. James, W. P. Martin, T. A. D. Smith, M. R. J. Elsegood, J. Chem. Soc., Perkin Trans. 1 2001, 3029-3036; d) S. M. Allin, S. L. James, W. P. Martin, T. A. D. Smith, Tetrahedron Lett. 2001, 42, 3943-3946; e) S. M. Allin, C. J. Northfield, M. I. Page, A. M. Z. Slawin, Tetrahedron Lett. 1998, 39, 4905-4908.

[9] See: S. M. Allin, C. I. Thomas, J. E. Allard, M. Duncton, M. R. J. Elsegood, M. Edgar, Tetrahedron Lett. 2004, 45, 7103-7105. Crystallographic data (excluding structure factors) for structures $5 \mathrm{a}(\mathrm{R}=0.072)$ and $6(\mathrm{R}=0.031)$ in this paper have been deposited with the Cambridge Crystallographic Data Centre (CCDC 227217-227218).

[10] M. Amat, J. Bosch, J. Hidalgo, M. Canto, M. Perez, N. Llor, E. Molins, C. Miravitlles, M. Orozco, J. Luque, J. Org. Chem. 2000, 65, 3074-3084.

[11] H. Akimoto, K. Okamura, M. Yui, T. Shioiri, M. Kuramoto, Y. Kikugawa, S. Yamada, Chem. Pharm. Bull. 1974, 22, 2614-2623.

[12] a) Compound $\mathbf{1 0}$ gave $[\alpha]_{\mathrm{D}}{ }^{20}=-286\left(c=1, \mathrm{CHCl}_{3}\right)$. The same compound is quoted as having $[\alpha]_{\mathrm{D}}{ }^{20}=-232\left(c=1.02, \mathrm{CHCl}_{3}\right)$ at an e.e. of $96 \%{ }^{13 \mathrm{a}}$; b) Compound 11: $[\alpha]_{\mathrm{D}}{ }^{20}=-$ $79.6(c=1, \mathrm{MeOH}) ;$ lit. $^{13 \mathrm{a}}:[\alpha]_{\mathrm{D}}{ }^{20}=-84(c=1, \mathrm{MeOH})$.

[13] a) A. I. Meyers, T. Sohda, M. F. Loewe, J. Org. Chem. 1986, 51, 3108-3112; b) T. Itoh, Y. Matsuya, Y. Enomoto, A. Ohsawa, Heterocycles 2001, 55, 1165-1171; c) L. Calabi, B. Danieli, G. Lesma, G. Palmisana, Tetrahedron Lett. 1982, 23, 2139-2142; d) L. E. Overman, T. C. Malone, J. Org. Chem. 1982, 47, 5297-5300; e) D. Thielke, J. Wegener, E. Winterfeldt, Chem. Ber. 1975, 108, 1791-1802; f) N. Sydorenko, C. A. Zificsak, A. I. Gerasyuto, R. P. Hsung, Org. Biomol. Chem. 2005, 3, 2140-2144. 
[14] The $E$ stereoisomer was found to display a chemical shift of $\delta 7.0-7.05 \mathrm{ppm}$ for the olefinic proton, whereas the $Z$ stereoisomer displayed the olefinic proton in a more upfield position of $\delta 5.92-5.97 \mathrm{ppm}$. This pattern is in line with reports of NMR data of geometrical isomers of similar indolo[2,3-a]quinolizidines. ${ }^{[17]}$ Compound $\mathbf{1 2}$ was ultimately converted to target $\mathbf{1 4}$, for which structural confirmation was obtained by X-ray crystallography, thus confirming the $E$ stereochemistry of both the final product and its precursors.

[15] S. Routier, L. Sauge, N. Ayerbe, G. Coudert, J.-Y. Merour, Tetrahedron Lett. 2002, 43, 589-591.

[16] S. F. Martin, K. X. Chen, C. T. Early, Org. Lett. 1999, 1, 79-81.

[17] B. J. Banks, M. J. Calverley, P. D. Edwards, J. Harley-Mason, Tetrahedron Lett. 1981, 22, 1631-1634. 